\title{
The D-CTC Condition is Generically Fulfilled in Classical (Non-quantum) Statistical Systems
}

\author{
Jürgen Tolksdorf ${ }^{1} \cdot$ Rainer Verch $^{1}$ (]
}

Received: 2 June 2021 / Accepted: 31 August 2021 / Published online: 23 September 2021

(C) The Author(s) 2021

\begin{abstract}
The D-CTC condition, introduced by David Deutsch as a condition to be fulfilled by analogues for processes of quantum systems in the presence of closed timelike curves, is investigated for classical statistical (non-quantum) bi-partite systems. It is shown that the D-CTC condition can generically be fulfilled in classical statistical systems, under very general, model-independent conditions. The central property used is the convexity and completeness of the state space that allows it to generalize Deutsch's original proof for q-bit systems to more general classes of statistically described systems. The results demonstrate that the D-CTC condition, or the conditions under which it can be fulfilled, is not characteristic of, or dependent on, the quantum nature of a bi-partite system.
\end{abstract}

Keywords Closed timelike curves $\cdot$ Classical statistical systems $\cdot$ Ergodicity

\section{Introduction}

In a seminal paper [1], David Deutsch introduced a condition (henceforth referred to as $D$-CTC condition) that is supposed to capture the meaning of processes "running back in time" in bi-partite quantum systems (and more generally, in multi-partite quantum systems, or quantum circuits). In its simplest form, it can be described as follows: Assume a bi-partite quantum mechanical system given, consisting of a Hilbert space $\mathcal{H}=\mathcal{H}_{A} \otimes \mathcal{H}_{B}$ composed of the Hilbert spaces of two subsystems. Moreover, suppose that $U$ is a unitary operator on $\mathcal{H}$, which is viewed as describing (the result of) a dynamical interaction between the two systems, akin to a time evolution

\footnotetext{
Communicated by Klaas Landsman.

Rainer Verch

rainer.verch@uni-leipzig.de

Jürgen Tolksdorf

juergen.tolksdorf@uni-leipzig.de

1 Institute for Theoretical Physics, University of Leipzig, 04009 Leipzig, Germany
} 
operator, or a scattering matrix. Furthermore, let $\varrho_{A}$ be a density matrix on $\mathcal{H}_{A}$, or synonymously, a state on the "A"-part of the system with expectation values

$$
\langle\mathbf{a}\rangle_{A}=\operatorname{Tr}_{A}\left(\varrho_{A} \mathbf{a}\right)
$$

for all bounded linear operators a on $\mathcal{H}_{A}$ where we have written $\operatorname{Tr}_{A}$ to emphasize that the trace is to be understood with respect to the Hilbert space $\mathcal{H}_{A}$. Relative to these data, a density matrix $\rho$ on $\mathcal{H}$ is said to fulfill the D-CTC condition if the following two conditions are fulfilled (see Fig. 1):

(1) The partial state induced by $\varrho$ on the "A"-part of the system (prior to the interaction $U$ taking effect) equals $\langle.\rangle_{A}$, i.e.

$$
\operatorname{Tr}\left(\rho\left(\mathbf{a} \otimes \mathbf{1}_{B}\right)\right)=\operatorname{Tr}_{A}\left(\varrho_{A} \mathbf{a}\right)
$$

holds for all bounded linear operators a on $\mathcal{H}_{A}$ (with $\mathbf{1}_{B}$ denoting the identity operator on $\mathcal{H}_{B}$ ). Equivalently, $\varrho_{A}$ equals $\operatorname{tr}^{\mathcal{H}_{B}}(\varrho)$, the partial trace of $\rho$ taken with respect to $\mathcal{H}_{B}$. Note that in the previous equation, the trace $\mathrm{Tr}$ appearing on the left hand side is taken on the full Hilbert space $\mathcal{H}=\mathcal{H}_{A} \otimes \mathcal{H}_{B}$.

(2) The partial state induced by $\varrho$ on the " $B$ "-part of the system (prior to the interaction $U$ ) returns to itself after the interaction $U$ has taken effect, i.e.

$$
\operatorname{Tr}\left(\rho U^{*}\left(\mathbf{1}_{A} \otimes \mathbf{b}\right) U\right)=\operatorname{Tr}\left(\varrho\left(\mathbf{1}_{A} \otimes \mathbf{b}\right)\right)
$$

holds for all bounded linear operators $\mathbf{b}$ on $\mathcal{H}_{B}$ (with $\mathbf{1}_{A}$ now denoting the identity operator on $\mathcal{H}_{A}$ ).

In his proof that the D-CTC condition can always be fulfilled when the Hilbert spaces $\mathcal{H}_{A}$ and $\mathcal{H}_{B}$ are both finite-dimensional, Deutsch uses that the map

$$
\mathcal{S}: \varrho_{B} \mapsto \operatorname{tr}^{\mathcal{H}_{A}}\left(U\left(\varrho_{A} \otimes \varrho_{B}\right) U^{*}\right)
$$

Fig. 1 A process in a quantum circuit is represented a unitary operator $U$ describing the dynamical coupling of two system parts (denoted by $A$ and $B)$. $U$ takes initial states (prior to interaction) to final states (after the interaction has taken effect); that process is supposed to take a time duration $T$. A "step backward in time" is symbolized by $-T$; the $B$-part of the result of the process (i.e. the partial state on the $B$ system after the interaction) is again fed into the process as initial state of the $B$-part

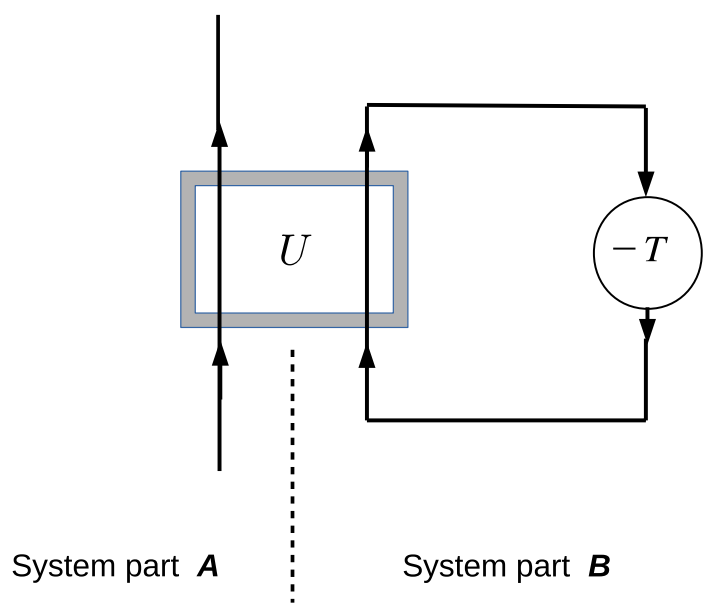


on the set of density matrices $\varrho_{B}$ on $\mathcal{H}_{B}$ has a fixed point. However, what is actually being used (and allows the fixed point argument to be applied) is that for quantum mechanical systems, the state space is always convex and complete: It allows for classical statistical (or probabilistic) mixtures of states, and limits (in a suitable sense) thereof. In other words, the D-CTC condition, and the question to which extent it can be fulfilled, is not primarily sensitive to, or dependent on, genuinely quantum mechanical properties of a bi-partite system, such as quantum mechanical superpositions (interference effects), uncertainty relations or entanglement, but really on the convexity and completeness of the state space of the systems in question. Therefore, the D-CTC condition can also be fulfilled in classical (i.e. non-quantum) statistical physical theories, such as classical statistical mechanics, under very general, physically realistic conditions; it is the purpose of this article to demonstrate that fact at an appreciable level of mathematical generality and rigour. The authors of [2] reach at a related conclusion, however based on a different reasoning than presented in this article; they argue that in the limit of large Hilbert space dimension of the "B" system part, the D-CTC condition becomes classical. In [3], the authors indicate that the D-CTC condition can be staged in a far more general formal framework than that of quantum mechanics. The feature of the D-CTC condition to be primarily dependent on the ability to form classical statistical mixtures of states has also been observed in [4]. That same article also discusses related investigations of classical "billiard ball" collisions wherein one of the balls enters a "wormhole"-type time machine and re-emerges "prior to entering" exactly such as to be kicked by the other ball into the time machine [5-7]. Such scenarios may be viewed as particular classical counterparts of the D-CTC set-up (or rather-historically more correctly - the D-CTC approach ought to be seen as an attempt at providing a quantum analogue for such "billiard-ball-collisions-with-wormhole-timemachines" set-ups) but we will not follow this line of analogy in the present article. See, however, Sect. 5 for further remarks.

The D-CTC condition is always presented in the context of quantum physics ${ }^{1}$ or of quantum computational considerations (as a sample, see the publications $[1,2$, 10-15], see also references therein). Therefore, it seems well worth pointing out, and demonstrating, that it is basically of a statistical, but not necessarily quantum physical nature.

In a recent paper [16] (see also [17] for a summary), we have investigated the D-CTC condition in the setting of operator-algebraic quantum field theory [18]. It is useful to briefly outline some of the basic elements of that approach as it helps to make parallels between the D-CTC condition as formulated above for quantum

\footnotetext{
1 The article [8] portrays quantum mechanics as a natural mechanism for avoiding paradoxes that would occur in the presence of CTCs in the framework of classical physics; however in [4] it is pointed out that forming statistical mixtures would in a similar way allow it to avoid those paradoxes. Nevertheless, linking the D-CTC condition with quantum physics has become a commonplace because of its origins and potential consequences in quantum computing (see references cited above), and seems to have also gained traction in popular culture: In [9], the lead character Tony Stark verbally mentions "the Deutsch proposition" in an attempt of the protagonists to travel back to the past using a fictional time machine based on quantum physics.
} 
mechanical systems, and the classical statistical physics case to be considered in this article, more easily visible.

In the operator-algebraic approach to relativistic quantum field theory [18], there is for any system (quantum field) a $C^{*}$-algebra $\mathcal{A}$ whose self-adjoint elements correspond to observables of the system. In most cases, it is no major restriction to suppose that $\mathcal{A}$ is a subalgebra of some $\mathrm{B}(\mathcal{H})$, the algebra of all bounded operators on an (infinite dimensional) Hilbert space $\mathcal{H}$. It is also usually assumed that $\mathcal{A}$ contains an algebraic unit element denoted by $\mathbf{1}$; if $\mathcal{A} \subset \mathrm{B}(\mathcal{H})$, that would be the unit operator on $\mathcal{H}$. Supposing that the spacetime on which the quantum field propagates is Minkowski spacetime (however, the general setting allows for choosing more general, curved spacetimes instead), it is assumed that for any finite (that is, relatively compact) open region $O$ in spacetime there is a $C^{*}$ subalgebra $\mathcal{A}(O)$ of $\mathcal{A}$ containing the observables that can be measured at times and locations within $O$, including 1. In keeping with this set-up, it is further assumed that $\mathcal{A}\left(O_{1}\right) \subset \mathcal{A}\left(O_{2}\right)$ whenever $O_{1} \subset O_{2}$. This property is called isotony. Another assumption is locality, meaning that $\mathbf{a b}=\mathbf{b a}$ for all $\mathbf{a} \in \mathcal{A}\left(O_{A}\right)$ and $\mathbf{b} \in \mathcal{A}\left(O_{B}\right)$ provided that the spacetime regions $O_{A}$ and $O_{B}$ are causally separated, i.e. there is no causal curve beginning in $O_{A}$ and ending $O_{B}$. Particularly in this situation where $O_{A}$ and $O_{B}$ are causally separated, one may take the pair of algebras $\mathcal{A}\left(O_{A}\right)$ and $\mathcal{A}\left(O_{B}\right)$ as the mathematical model of a causally separated bi-partite system, with $\mathcal{A}\left(O_{A}\right)$ and $\mathcal{A}\left(O_{B}\right)$ playing roles analogous to $\mathrm{B}\left(\mathcal{H}_{A}\right)$ and $\mathrm{B}\left(\mathcal{H}_{B}\right)$ in the quantum mechanical setting outlined at the beginning.

Another important ingredient of the operator-algebraic approach are states. A state is any expectation value functional $\mathbf{a} \mapsto\langle\mathbf{a}\rangle \quad(\mathbf{a} \in \mathcal{A})$ on the algebra of observables $\mathcal{A}$, and therefore, by definition, $\mathbf{a} \mapsto\langle\mathbf{a}\rangle$ is linear, and fulfills $\left\langle\mathbf{a}^{*} \mathbf{a}\right\rangle \geq 0$ for all $\mathbf{a} \in \mathcal{A}$, as well as $\langle\mathbf{1}\rangle=1$. Usually, if $\mathcal{A}$ is contained in some $\mathrm{B}(\mathcal{H})$, one considers only normal states which arise from density matrices; in other words, a state is normal if it is of the form

$$
\langle\mathbf{a}\rangle=\langle\mathbf{a}\rangle_{\rho}=\operatorname{Tr}(\varrho \mathbf{a}) \quad(\mathbf{a} \in \mathcal{A})
$$

for some density matrix $\varrho$ on the Hilbert space $\mathcal{H}$.

One may now reformulate the D-CTC condition in the operator-algebraic setting as follows. As mentioned, one starts from an observable algebra $\mathcal{A} \subset \mathrm{B}(\mathcal{H})$ for some Hilbert space $\mathcal{H}$, together with observable algebras $\mathcal{A}\left(O_{A}\right)$ and $\mathcal{A}\left(O_{B}\right)$ for two causally separated spacetime regions $O_{A}$ and $O_{B}$, representing the observables of a causally separated bi-partite system. Further data assumed given are a normal state $\langle\mathbf{a}\rangle_{A}=\operatorname{Tr}\left(\varrho_{A} \mathbf{a}\right) \quad\left(\mathbf{a} \in \mathcal{A}\left(O_{A}\right)\right)$ on $\mathcal{A}\left(O_{A}\right)$ (on the "A"-part of the full system) induced by a density matrix $\varrho_{A}$ on $\mathcal{H}$, and a unitary operator $U$ on $\mathcal{H}$. Given these data, a state $\langle\mathbf{c}\rangle=\operatorname{Tr}(\rho \mathbf{c}) \quad(\mathbf{c} \in \mathcal{A})$ is said to fulfill the D-CTC condition if the following two conditions are fulfilled:

(I) The partial state of $\langle$.$\rangle on \mathcal{A}\left(O_{A}\right)$ coincides with $\langle.\rangle_{A}$, i.e.

$$
\langle\mathbf{a}\rangle=\langle\mathbf{a}\rangle_{A} \quad\left(\mathbf{a} \in \mathcal{A}\left(O_{A}\right)\right)
$$


(II) The partial state of $\langle$.$\rangle on \mathcal{A}\left(O_{B}\right)$ returns to itself after the action of the unitary $U$ has taken effect, i.e.

$$
\left\langle U^{*} \mathbf{b} U\right\rangle=\langle\mathbf{b}\rangle \quad\left(\mathbf{b} \in \mathcal{A}\left(O_{B}\right)\right)
$$

The analogy with the D-CTC condition with the quantum mechanical case described above should be clear on noting that, since both $\mathcal{A}\left(O_{A}\right)$ and $\mathcal{A}\left(O_{B}\right)$ are in a defined way subalgebras of the larger $C^{*}$-algebra $\mathcal{A}$ (or of $\mathrm{B}(\mathcal{H})$ ), and all the algebras share the common algebraic unit element $\mathbf{1}$, the $\mathbf{a} \in \mathcal{A}\left(O_{A}\right)$ here is analogous to the $\mathbf{a} \otimes \mathbf{1}$ above, and similarly the $\mathbf{b} \in \mathcal{A}\left(O_{B}\right)$ here is analogous to the $\mathbf{1} \otimes \mathbf{b}$ above. We mention however that in general, in quantum field theory the operator algebra generated by $\mathcal{A}\left(O_{A}\right)$ and $\mathcal{A}\left(O_{B}\right)$ in $\mathrm{B}(\mathcal{H})$ need not equal (up to identification) the tensor product $\mathcal{A}\left(O_{A}\right) \otimes \mathcal{A}\left(O_{B}\right)$. A precise statement would require introducing von Neumann algebras at this point which we shall not embark on. Nevertheless, there are criteria as to when such an equality actually does hold, known as split property or statistical independence of states. We will not further discuss these matters here but refer to [18-20] and references cited there for full details.

The results obtained in $[16,17]$ are, roughly, as follows (we give here a mainly qualitative description and refer to the cited references for full details). States fulfilling the D-CTC condition cannot be found if the states are also required to fulfill a ReehSchlieder like property [18] which implies a strong form of entanglement [21]. On the other hand, if the local algebras of observables fulfill the split property just mentioned, then one can always find states fulfilling the D-CTC condition approximately to any prescribed precision. Since the assumptions are met for a wide range of quantum field theories on globally hyperbolic spacetimes which do not admit closed timelike curves, the latter result makes it doubtful if the D-CTC condition actually relates to quantum processes based on the presence of closed timelike curves in the sense of general relativity. The present work casts doubts on whether the D-CTC condition has quantum physics at its core. We will address these points in a discussion towards the end in Sect. 5 .

We now turn to describing the content of the present work. In Sect. 2, we will summarize some basics of commutative $C^{*}$-algebras which, in an operator algebraic approach, are used as algebras of observables of classical (non-quantum) statistical systems. The relation to functions (random variables) on locally compact or compact topological Hausdorff spaces and probability measures (states) - through the Riesz Representation Theorem and the Gelfand-Naimark Theorem-is also discussed. We have relied on the references [22-25] for our presentation which, on one hand, is included to make this work self-contained and to introduce the concepts and notation needed, and on the other hand, to explain some points that need to be taken care of when considering limits of states on certain commutative $C^{*}$-algebras and the question if they still arise from probability measures. We take up on this topic again in Sect. 3 where the concept of classical statistical bi-partite systems is introduced. A criterion ensuring that limits of sequences of probability measures exist on $C_{b}(X)$, the $C^{*}$-algebra of bounded continuous functions on a locally compact metric space $X$, and are again probability measures, is provided by Prohorov's Theorem [26, 27] and we use it in Theorem 3.2. In our Theorem 3.1 presented before in Sect. 3, we 
prove a very general statement to the effect that the D-CTC condition for classical statistical bi-partite systems is fulfilled but with states in an abstract $C^{*}$-algebraic sense which need not be given by probability measures. As indicated, Theorem 3.2 is more specific in that it establishes that the states fulfilling the D-CTC condition are given by probability measures under certain assumptions. A simple example in form of a two-body problem interacting by a binding central potential is discussed in Sect. 4 to illustrate properties of the states fulfilling the D-CTC condition constructed in Theorems 3.1 and 3.2. The example will also serve to point out a relation to ergodicity. In the last section, we collect discussion and conclusion, relating our results also to other literature.

\section{Commutative $C^{*}$-Algebras and Classical Statistical Systems}

\subsection{Generalities}

Physical systems that are subject to a statistical description of their measurement values, but are classical in the sense of not being quantum systems, have observable algebras which are commutative (or Abelian). Let us denote a generic commutative $C^{*}$ -algebra by $A$. Commutativity means that $f g=g f$ for all $f, g \in A$ and consequently, there are no uncertainty relations among the elements of $A$ which would be indicative of a quantum theory. Likewise, there is no entanglement. Assuming that there is a unit element 1 contained in $A$, a state on $A$ is defined as a linear functional $\langle\rangle:. A \rightarrow \mathbb{C}$, $f \mapsto\langle f\rangle$ fulfilling $\left\langle f^{*} f\right\rangle \geq 0$ (positivity) and $\langle 1\rangle=1$ (normalization). We recall the the well-known fact that any state $w$ on a $C^{*}$-algebra (commutative or not) is norm-continuous: $|w(\mathbf{f})| \leq\|\mathrm{f}\|$ for all $\mathrm{f}$ where $\|\mathrm{f}\|$ is the $C^{*}$-algebra norm of $\mathrm{f}[24]$. It is worth mentioning that $C^{*}$-algebras are algebras over $\mathbb{C}$ (as field of numbers) but that, as in quantum mechanics, only their hermitean elements, fulfilling $f^{*}=f$, are considered as observables yielding real-valued expectation values $w(f)=\langle f\rangle$ upon evaluation on states.

We shall now adopt the mathematical notation and denote a state as $w: A \rightarrow \mathbb{C}$ so that $w(f)=\langle f\rangle(f \in A)$, since this notation has some advantages. It is easy to notice that the set of states on $\mathrm{A}$, henceforth denoted as $\mathscr{S}=\mathrm{A}_{+}^{*}$, is closed under finite convex combinations, i.e. if $w_{1}, \ldots, w_{n}$ are finitely many states on $\mathrm{A}$ and $\lambda_{1}, \ldots, \lambda_{n}$ are nonnegative numbers such that $\sum_{k=1}^{n} \lambda_{k}=1$, then the convex sum $\sum_{k=1}^{n} \lambda_{k} w_{k}$ that can be formed from the given states is again a state on A. A state is called pure if it can be represented in this convex sum form if, and only if, all the $w_{k}$ coincide; or equivalently, iff all $\lambda_{k}=0$ except for exactly one $\lambda_{k^{\prime}}$ which therefore must be equal to 1 . A state which is not pure is called mixed. Furthermore, the set of states is closed with respect to taking weak limits: Suppose that $\left\{w_{\kappa}\right\}_{\kappa \in \mathcal{K}}$ is a generalized sequence of states $w_{\kappa} \in \mathscr{S}$, where $\mathcal{K}$ is an arbitrary directed set. The generalized sequence $\left\{w_{K}\right\}_{\kappa \in \mathcal{K}}$ is called weakly convergent (strictly speaking, weak-*-convergent) if $\lim _{\kappa} w_{\kappa}(\mathrm{f})$ exists for every $\mathrm{f} \in \mathrm{A}$. Then

$$
w(\mathbf{f})=\lim _{\kappa} w_{\kappa}(\mathbf{f}) \quad(\mathrm{f} \in \mathbf{A})
$$


is again a state on $\mathrm{A}$. We mention also that $\mathscr{S}$ is weakly compact by the Banach-Alaoglu-Theorem [28], which entails that, whenever $\left\{w_{\kappa}\right\}_{\kappa \in \mathcal{K}}$ is a generalized sequence in $\mathscr{S}$, then it admits a weakly convergent generalized subsequence $\left\{w_{\kappa(\zeta)}\right\}_{\zeta \in \mathcal{Z}}$ (with suitable directed index set $\mathcal{Z}$ ).

An operation is any map $\tau: \mathscr{S} \rightarrow \mathscr{S}$ which preserves convexity, meaning that

$$
\tau\left(\sum_{k=1}^{n} \lambda_{k} w_{k}\right)=\sum_{k=1}^{n} \lambda_{k} \tau\left(w_{k}\right)
$$

for all finite convex sums of states. Moreover, we will assume operations to be weakly continuous ${ }^{2}$ which is defined as follows: $\tau$ is weakly continuous if, for all weakly converging generalized sequences $\left\{w_{\kappa}\right\}_{\kappa \in \mathcal{K}}$ of states in $\mathscr{S}$, also $\left\{\tau\left(w_{K}\right)\right\}_{\kappa \in \mathcal{K}}$ is a weakly converging generalized sequence of states, with $\lim _{\kappa} \tau\left(w_{\kappa}\right)(\mathrm{f})=\tau\left(\lim _{\kappa} w_{\kappa}\right)(\mathrm{f})$ for all $\mathrm{f} \in \mathrm{A}$. We will see some examples soon; obviously, if $\alpha: \mathrm{A} \rightarrow \mathrm{A}$ is a $C^{*}$-algebra morphism which preserves the unit element, then its dual map $\alpha^{*}(w)(\mathbf{f})=w(\alpha(\mathbf{f}))$ is an operation.

\subsection{The Gelfand-Naimark Theorem}

The next step is to summarize the content of the Gelfand-Naimark theorem (see Lemma 2 in [29]) which characterizes commutative $C^{*}$-algebras as sets of numbervalued functions and the states as probability measures. To this end, we largely follow the presentations of [22-24] which we recommend for further reading.

Let $\mathrm{A}$ denote a commutative $C^{*}$-algebra with unit element 1 . Then the Gelfand-Naimark theorem asserts that there is a compact topological Hausdorff space $X$ and a $C^{*}$-algebra isomorphism $\phi: \mathrm{A} \rightarrow C^{0}(X)$ which preserves the unit. Here, $C^{0}(X)$ is the vector space of all continuous functions on $X$ taking values in $\mathbb{C}$; endowing it with the pointwise product $(f g)(x)=f(x) g(x)(x \in X)$ as an algebra product and complex conjugation as the *-operation, and taking as $C^{*}$-norm $\|f\|_{\infty}=\sup _{x \in X}|f(x)|$, $C^{0}(X)$ is a commutative $C^{*}$-algebra. Its unit element clearly is the function $1(x)=1$ $(x \in X)$ taking identically the value 1 . Moreover, for any state $w$ on $\mathrm{A}$, the induced state $w^{\phi}(f)=w\left(\phi^{-1}(f)\right)$ on $C^{0}(X)$ is given by a probability measure $\mu_{w}$ defined on the Borel sets of $X$ :

$$
w^{\phi}(f)=\int_{X} f(x) d \mu_{w}(x) \quad\left(f \in C^{0}(X)\right) .
$$

A probability measure is normalized so that $\int_{X} 1 d \mu_{w}=1$. Furthermore, a state $w$ on $\mathrm{A}$ is pure if and only if the measure $\mu_{w}$ is concentrated at a single point $x_{0}$ in $X$ (a "Dirac measure"), that is, $w^{\phi}(f)=f\left(x_{0}\right)$ for all $f \in C^{0}(X)$. Therefore, the Gelfand transform $\mathrm{f} \mapsto f, f(x)=w_{x}(\mathrm{f})$, where the $w_{x}(x \in X)$ range over the set of pure states on $\mathrm{A}$, provides the concrete realization of the isomorphism $\phi$. Any homeomorphism $F: X \rightarrow X$ gives rise to a $C^{*}$-isomorphism $A_{F}: C^{0}(X) \rightarrow C^{0}(X)$ given

\footnotetext{
${ }^{2}$ Strictly speaking, the continuity property defined here is weak-*-continuity.
} 
by $A_{F}(f)=f \circ F^{-1}$ and one has $A_{F}(1)=1$; pulling $A_{F}$ back by $\phi$ renders a $C^{*}$-algebra isomorphism $\alpha_{F}$ of A given by $\alpha_{F}=\phi^{-1} \circ A_{F} \circ \phi$ which preserves the unit element 1. Consequently, the dual map $\tau_{F}=\alpha_{F}^{*}$ is an operation on the set of states on A. On the Borel measures of $X$, this operation is given as $\mu \mapsto A_{F}^{*}(\mu)=\mu \circ F$ which can be seen from the measure-transformation equation (cf. [25, Theorem 12.46])

$$
\int_{X} f \circ F^{-1} d \mu=\int_{X} f d(\mu \circ F) \quad\left(f \in C^{0}(X)\right)
$$

Consequently, for a commutative $C^{*}$-algebra, operations on the set of states arise from bijective homeomorphisms in the indicated way. There are also operations typically not arising in this way. A simple example is $\tau: w \mapsto \frac{1}{2}\left(w_{0}+w\right)$ where $w_{0}$ is any fixed but arbitrary state. Another class of examples concerns operations on a particular set of states. Assume that a commutative $C^{*}$-algebra with unit element is given as $C^{0}(X)$ for a compact Hausdorff space $X$, and select any state $w_{0}$, i.e. a probability measure $\mu_{0}$ on the Borel sets of $X$. Then the Hilbert space of the GNS representation (see Theorem 1 in [29], or Theorem 3.3.3 in [24] for a more modern version $)^{3}$ is given as $L^{2}\left(X, \mu_{0}\right)$ where at this point, one should bear in mind that the $L^{2}$ space is formed by equivalence classes of square-integrable functions on $X$ where functions are defined as equivalent iff they deviate on sets of zero $\mu_{0}$ measure. With respect to the chosen $\mu_{0}$, one can introduce normal states $w_{\varrho}(f)=\operatorname{Tr}(\varrho f)\left(f \in C^{0}(X)\right)$ where $\rho$ is any density matrix on the Hilbert space $L^{2}\left(X, \mu_{0}\right)$ and $f \in C^{0}(X)$ acts as multiplication operator on $L^{2}\left(X, \mu_{0}\right)$. Then any unitary linear operator $U$ on $L^{2}\left(X, \mu_{0}\right)$ induces the operation $\tau_{U}: w_{\rho} \mapsto w_{U \rho U^{*}}$ on the set of normal states with respect to $\mu_{0}$ ${ }^{4}$ As a side note, a formulation of classical (statistical) mechanics in a related $L^{2}$ -space setting appears in [30]; it also serves as a starting point in the so-called geometric quantization [31].

\subsection{The Case of $A=C_{b}(X)$ for $X$ Non-compact}

The discussion up to now should have clarified the bijective relation between communtative $C^{*}$-algebras with unit element and their states, and the algebras $C^{0}(X)$ on compact Hausdorff spaces $X$ and the probability measures on the corresponding Borel sets. The latter mathematical framework is the starting point of classical statistical theories. Here, $X$ usually contains the (classical) degrees of freedom of a physical system; in fact, most commonly $X=T^{*} Q$ is the phase space of a system whose degrees of freedom can move in some smooth manifold $Q$. In this situation, there arises the difficulty that even if $Q$ happens to be compact (which needn't be the case), $T^{*} Q$ is not. Therefore, we are confronted with the circumstance that in

\footnotetext{
${ }^{3}$ Given any $C^{*}$-algebra $\mathcal{A}$ (not necessarily commutative) containing a unit element, and a state $\omega$ on $\mathcal{A}$, the GNS representation is a triple $(\mathcal{H}, \pi, \Omega)$ where $\mathcal{H}$ is a Hilbert space, $\pi$ is a unital *-representation of $\mathcal{A}$ by bounded linear operators on $\mathcal{H}$ and $\Omega$ is a unit vector in $\mathcal{H}$ so that $\omega(\mathbf{a})=\langle\Omega, \pi(\mathbf{a}) \Omega\rangle$ holds for all $\mathbf{a} \in \mathcal{A}$, and $\pi(\mathcal{A}) \Omega$ is dense in $\mathcal{H}$. For every state on a unital $C^{*}$-algebra there is such a GNS representation and it is unique up to unitary equivalence.

4 Such an operation is in general only weakly sequentially continuous.
} 
many physically relevant cases, $X$ isn't in a natural way compact. This issue is of some concern for us because it has some consequences for the convergence of states which we need to consider in order to obtain solutions to the D-CTC problem in the following section.

Therefore, assume now that $X$ is a locally compact Hausdorff topological space, and define $C_{0}(X)$ as the set of all continuous functions $f: X \rightarrow \mathbb{C}$ that vanish at infinity, i.e. given $f \in C_{0}(X)$, there is for every $\varepsilon>0$ some compact set $K$ such that $|f(x)|<\varepsilon$ for all $x \in X \backslash K$. Using the same definitions for the algebraic operations as for $C^{0}(X)$, the set $C_{0}(X)$ is a commutative $C^{*}$-algebra with $C^{*}$-norm $\|f\|_{\infty}=\sup \{|f(x)|: x \in X\}$. If $X$ is compact, then $C_{0}(X)=C^{0}(X)$ (cf. [25, Sect. (7.13a)]), but if $X$ is not compact, then $C_{0}(X)$ is a commutative $C^{*}$-algebra without an algebraic unit element. One can still define probability measures $\mu$ on the Borel sets of $X$ as the Borel measures that have unit weight, $\mu(X)=1$. This is equivalent to requiring that the positive functional $w(f)=\int_{X} f d \mu\left(f \in C_{0}(X)\right)$ induced by $\mu$ on $C_{0}(X)$ has unit norm, that is, $\|w\|=1$ where $\|w\|=\sup \left\{|w(f)|:\|f\|_{\infty}=1\right\}$.

The Gelfand-Naimark theorem which has been stated above for the case of a commutative $C^{*}$-algebra $\mathrm{A}$ with an algebraic unit element has the following extension to the case that A doesn't possess an algebraic unit element: There is a locally compact Hausdorff space $X$ and a $C^{*}$-algebraic isomorphism $\phi: \mathrm{A} \rightarrow C_{0}(X)$ which again is given by the Gelfand transform; so any state $w$ on $\mathrm{A}$ (where the normalization condition, in absence of the algebraic unit 1, is replaced by the condition that $w$ has unit norm, $\|w\|=1)$ induces a state $w^{\phi}(f)=w\left(\phi^{-1}(f)\right)\left(f \in C_{0}(X)\right)$ on $C_{0}(X)$ which is given by a probability measure on the Borel sets of $X$ (this is exactly the statement of the Riesz' Representation Theorem, see e.g. [25, Theorem 12.36]), and the pure states on $A$ are exactly those which arise as probability measures concentrated at single points of $X$.

For any locally compact Hausdorff space $X, C_{0}(X)$ is naturally a $C^{*}$-subalgebra of $C_{b}(X)$, the set of all bounded continuous functions $f: X \rightarrow \mathbb{C}$. Clearly, $C_{b}(X)$ becomes a $C^{*}$-algebra using the analogous algebraic operations as defined previously for $C_{0}(X)$, and again, $\|f\|_{\infty}=\sup \{|f(x)|: x \in X\}$ as $C^{*}$-norm. However, $C_{b}(X)$ contains an algebraic unit element given by the function taking the constant value 1 , similarly as for $C^{0}(X)$ for a compact $X$. While in the case that $X$ is not compact, $C_{0}(X)$ is a proper $C^{*}$-subalgebra of $C_{b}(X)$, any state $w$ on $C_{0}(X)$, by being induced by a probability measure on the Borel sets of $X$, extends uniquely to a state on $C_{b}(X)$, complying with the normalization condition $w(1)=1$. Since $C_{b}(X)$ is a commutative $C^{*}$-algebra with an algebraic unit element, by the Gelfand-Naimark theorem it is isomorphic to $C^{0}(\hat{X})$ for a particular compact Hausdorff space $\hat{X}$, the Stone- $\breve{C} e c h$ compactification of $X$. In fact, for any locally compact Hausdorff space $X$, "extending" $C_{0}(X)$ to $C_{b}(X)$ can be viewed as the "standard model" of the Stone-Čech compactification. We refer to the references [22-24] for further discussion and references on this point.

We therefore choose the commutative $C^{*}$-algebra $C_{b}(X)$ with a locally compact (but not necessarily compact) Hausdorff space $X$ as the most suitable and versatile version of an observable algebra for a classical statistical system since.

There are the following rationales for that choice: 
(i) As already mentioned, we view the phase space $T^{*} Q$ of a mechanical system as the standard example for $X$, and $T^{*} Q$ isn't compact in a natural way.

(ii) $C_{b}(X)$ contains an algebraic unit element while $C_{0}(X)$ does not for non-compact $X$. However, having a unit is important since it allows to approximate unbounded functions, which often represent important observables such as the Hamilton function $H$ (assumed continuous), by elements of $C_{b}(X)$ at the level of expectation values. Namely, in the presence of a unit 1, one can form the resolvents $\left(1+\epsilon H^{2}\right)^{-1}(\epsilon>0)$, and then the functions $H_{\epsilon}=\left(1+\epsilon H^{2}\right)^{-1} H$ are in $C_{b}(X)$ and for sufficiently regular states one obtains $\lim _{\epsilon \rightarrow 0} w\left(H_{\epsilon}\right)=w(H)$. Therefore, we see $C_{b}(X)$, possessing a unit element, as preferred since it allows the approximation of unbounded observables in a canonical way.

(iii) We wish to explore the D-CTC condition in the setting of classical probability theory where, by definition, the states are given by probability measures. As mentioned, any state on $C_{0}(X)$ is actually induced by a probability measure according to Riesz' theorem, and it extends to a state, induced by the same probability measure, on $C_{b}(X)$. Therefore, we are not missing any states by choosing $C_{b}(X)$ as observable algebra instead of $C_{0}(X)$.

It should be obvious that the operations on states considered previously for $C^{0}(X)$ with a compact $X$, in particular those induced by bijective homeomorphisms of $X$, have their completely analogous counterparts also in the case of $C_{b}(X)$ with locally compact $X$.

However, if $X$ is not compact, then there are states on the $C^{*}$-algebra $C_{b}(X)$ which are not given by probability measures on the Borel sets on $X$. Consider as a particular example the case $X=\mathbb{R}$, and the sequence of states on $C_{b}(\mathbb{R})$ given by $w_{n}(f)=f(n)(n \in \mathbb{N})$, i.e. the point-measures concentrated at the integers. If this sequence of states is restricted to $C_{0}(\mathbb{R})$, it converges for $n \rightarrow \infty$ to the zerofunctional, $\lim _{n \rightarrow \infty} w_{n}(f)=0$ for all $f \in C_{0}(\mathbb{R})$. Clearly, this functional is not induced by a probability measure and therefore the $w_{n}$ (or any generalized subsequence) do not converge weakly to a state on $C_{0}(\mathbb{R})$. On the other hand, by the Banach-Alaoglu Theorem mentioned before, there is a generalized subsequence $\{n(\kappa)\}_{\kappa \in \mathcal{K}}$ in $\mathbb{N}$ with $\lim _{\kappa} n(\kappa)=\infty$ so that the generalized subsequence $\left\{w_{n(\kappa)}\right\}_{\kappa \in \mathcal{K}}$ of states on $C_{b}(\mathbb{R})$ converges weakly to a state $w(f)=\lim _{\kappa} w_{n(\kappa)}(f)$. That state $w$ isn't induced by a probability measure on the Borel sets of $\mathbb{R}$ since $w(1)=1$ while $w(f)=0$ for all $f \in C_{0}(\mathbb{R})$. One may argue that such states have pathological properties and therefore aren't induced by probability measures and should not be regarded as physically realistic states. In order to make the distinction visible in the notation we will, for the commutative $C^{*}$-algebra $\mathrm{A}=C_{b}(X)$, denote the set of all $C^{*}$-algebraic states by $\mathscr{S}$ as before, and denote the set of states induced by probability measures by $\mathscr{S}^{(P)}$. If $X$ isn't compact, then $\mathscr{S}^{(P)}$ is a proper subset of $\mathscr{S}$.

The arguments leading to the results on the generic solvability of the D-CTC problem for classical bi-partite statistical systems that we shall derive in the next section make considerable use of the convergence of (generalized) sequences of states. Having made the point that we consider $C_{b}(X)$ with a possibly non-compact $X$ as algebra of observables, we would like to specify criteria ensuring that solutions 
to the D-CTC problem for classical bi-partite statistical systems are given by states which actually are induced by probability measures. As we will see in the next section, the condition of "tightness" on sequences of probability measures in combination with Prohorov's Theorem [26, 27] provide such criteria.

\section{Classical Statistical Bi-Partite Systems and the D-CTC Condition}

We define a classical statistical bi-partite system to consist of a direct product $X=X_{A} \times X_{B}$ where $X_{A}$ and $X_{B}$ are locally compact, Hausdorff topological spaces (or, for one of our results below, metric spaces). Then $X$ is also a locally compact Hausdorff space (res., metric space). We usually think of $X_{A}$ and $X_{B}$ as containing the degrees of freedom of two system parts labelled "A" and "B", e.g. $X_{A}=T^{*} Q_{A}$ and $X_{B}=T^{*} Q_{B}$ might be phase spaces over finite dimensional configuration manifolds of many particle systems. The system parts are independent but can be coupled dynamically. Then we take as observable algebras of the subsystems $\mathrm{A}_{A}=C_{b}\left(X_{A}\right)$ and $\mathrm{A}_{B}=C_{b}\left(X_{B}\right)$; the observable algebra of the full system will be $\mathrm{A}=C_{b}(X)$. Here, one can think of functions on phase space as the classical example. form

Then $\mathrm{A}=C_{b}(X)$ contains the $C^{*}$-subalgebra $\mathrm{A}_{\hat{\otimes}}$ generated by all elements $f$ of the

$$
f=\sum_{j=1}^{N} f_{j} \otimes g_{j}
$$

where $N \in \mathbb{N}$ and $f_{j} \in C_{b}\left(X_{A}\right)$ and $g_{j} \in C_{b}\left(X_{B}\right)$ where the tensor product is defined by $f_{j} \otimes g_{j}\left(x_{A}, x_{B}\right)=f_{j}\left(x_{A}\right) g_{j}\left(x_{B}\right)$ for all $x_{A} \in X_{A}, x_{B} \in X_{B}$. We will also write $\mathrm{A}_{\hat{\otimes}}=C_{b}\left(X_{A}\right) \hat{\otimes} C_{b}\left(X_{B}\right)$. Note that $\mathrm{A}_{\hat{\otimes}}$ contains the unit element of $\mathrm{A}=C_{b}(X)$.

If $w_{A}$ is a state on $C_{b}\left(X_{A}\right)$ and $w_{B}$ is a state on $C_{b}\left(X_{B}\right)$, then one can define the product state $w$ on $\mathrm{A}_{\hat{\otimes}}$ by setting

$$
w(f \otimes g)=w_{A}(f) w_{B}(g) \quad\left(f \in C_{b}\left(X_{A}\right), \quad g \in C_{b}\left(X_{B}\right)\right)
$$

and extension by linearity. In the case that $\mathrm{A}_{\hat{\otimes}}$ is a proper $C^{*}$-subalgebra of $C_{b}(X)$, one can still extend the product state $w$ to a state on $\mathrm{A}=C_{b}(X)$ ([24, Prop. 3.1.6]) which however need not be unique. We call any such state a product state extension of $w_{A}$ and $w_{B}$ (to $\left.\mathrm{A}=C_{b}(X)\right)$.

If $w_{A}$ and $w_{B}$ are states induced by probability measures $\mu_{A}$ and $\mu_{B}$ on the Borel sets of $X_{A}$ and $X_{B}$ respectively, then there is a unique product state $w$ induced by a unique probability measure $\mu=\mu_{A} \times \mu_{B}$, the product measure of $\mu_{A}$ and $\mu_{B}$, on the Borel sets of $X$. The product measure is determined by

$$
\left(\mu_{A} \times \mu_{B}\right)(O \times P)=\mu_{A}(O) \mu_{B}(P)
$$

for Borel sets $O$ of $X_{A}$ and $P$ of $X_{B}$ (see Sect. 21 in [25]). We now turn to our results establishing that the D-CTC condition can be fulfilled in classical statistical bipartite systems in great generality. In Theorem 3.1 we prove a statement to this end entirely set in the $C^{*}$-algebraic framework, where the states aren't necessarily 
induced by probability measures. Then we present another version in Theorem 3.2 where the states are induced by probability measures; it is for this result that we make use of Prohorov's Theorem, summarized below in this section. In the remark following the statement of Theorem 3.1, we explain how the formulation of the D-CTC condition given here connects to (and is, in fact, more general than) that of $[1,16]$.

Theorem 3.1 Let $X=X_{A} \times X_{B}$ define a classical statistical bi-partite system where $X_{A}$ and $X_{B}$ are locally compact, Hausdorff topological spaces. Let $\tau: \mathscr{S} \rightarrow \mathscr{S}$ be an operation and let $w_{A} \in \mathscr{S}_{A}$ be a state (in the $C^{*}$-algebraic sense) on $C_{b}\left(X_{A}\right)$.

Then there is a state $w \in \mathscr{S}$ on $C_{b}(X)$ (in the $C^{*}$-algebraic sense) with the properties

$$
\begin{aligned}
& w\left(f_{A} \otimes 1\right)=w_{A}\left(f_{A}\right) \quad\left(f_{A} \in C_{b}\left(X_{A}\right)\right) \quad \text { and } \\
& \tau(w)\left(1 \otimes f_{B}\right)=w\left(1 \otimes f_{B}\right) \quad\left(f_{B} \in C_{b}\left(X_{B}\right)\right) .
\end{aligned}
$$

Remark (3.1.A) In line with the terminology in [16], we say that a state $w$ with the properties (3.1) and (3.2) fulfills the D-CTC condition, or is a solution to the $D$-CTC problem, with respect to the given $X=X_{A} \times X_{B}, \tau$ and $w_{A}$.

(3.1.B) In Sect. 1-where the D-CTC condition of [1] has been summarizedand in [16], the operations are always induced by unitary operators on some Hilbert space on which the algebra of observables is represented; in other words, they are of the form $\tau(\langle\rangle)=.\left\langle U^{*} . U\right\rangle$ with a unitary operator $U$. The setting here is more general in that this assumption is not being made. In contrast, another assumption on operations which enters here is that the operations are assumed to be weakly continuous in the sense described above which is a natural assumption in the context of states on $C^{*}$-algebras. That is not always a natural assumption when operations are induced by unitary operators where usually weak sequential continuity is a more natural requirement. Theorem 3.2 below actually only requires weak sequential continuity of the operation $\tau$.

(3.1.C) If both $X_{A}$ and $X_{B}$ are compact, then the state $w$ is induced by a probability measure according to the Gelfand-Naimark theorem. One can deduce this also from Theorem 3.2, since the tightness assumptions entering in Theorem 3.2 are automatically fulfilled if both $X_{A}$ and $X_{B}$ are compact.

Proof of Theorem 3.1 The proof is analogous to the proof given in [16] in the operator-algebraic quantum field theory context, which in turn is based on the idea of the proof by Deutsch for the quantum mechanical case in finite-dimensional Hilbert spaces [1].

Choose any state $w_{B}^{\circ}$ in $\mathscr{S}_{B}$ and define the state $\varphi_{1}$ in $\mathscr{S}$ by choosing a product state extension of $w_{A}$ and $w_{B}^{\circ}$, thence obeying

$$
\varphi_{1}\left(f_{A} \otimes f_{B}\right)=w_{A}\left(f_{A}\right) w_{B}^{\circ}\left(f_{B}\right), \quad f_{A} \in C_{b}\left(X_{A}\right), f_{B} \in C_{b}\left(X_{B}\right) .
$$


Then define a sequence of states $\varphi_{n}(n \in \mathbb{N})$ in $\mathscr{S}$ inductively choosing product state extensions of $w_{A}$ and the partial state $f_{B} \mapsto \tau\left(\varphi_{n}\right)\left(1 \otimes f_{B}\right)$, so that

$$
\varphi_{n+1}\left(f_{A} \otimes f_{B}\right)=w_{A}\left(f_{A}\right) \cdot \tau\left(\varphi_{n}\right)\left(1 \otimes f_{B}\right) \quad(n \in \mathbb{N}) .
$$

Note that, as $\tau\left(\varphi_{n}\right)$ is in $\mathscr{S}$, the partial state $f_{B} \mapsto \tau\left(\varphi_{n}\right)\left(1 \otimes f_{B}\right)$ is in $\mathscr{S}_{B}$ which then implies that one may choose a product state extension $\varphi_{n+1}$ from $\mathrm{A}_{\hat{\otimes}}=C_{b}\left(X_{A}\right) \hat{\otimes} C_{b}\left(X_{B}\right)$ to $\mathrm{A}=C_{b}(X)$. Without additional conditions however, the product state extensions might be non-unique. The definition of the $\varphi_{n}$ implies for all $n \in \mathbb{N}$ (notwithstanding their potential non-unique extension to $\mathrm{A}=C_{b}(X)$ ),

$$
\begin{aligned}
\varphi_{n+1}\left(f_{A} \otimes 1\right) & =w_{A}\left(f_{A}\right) \cdot \tau\left(\varphi_{n}\right)(1 \otimes 1)=w_{A}\left(f_{A}\right), \\
\varphi_{1}\left(f_{A} \otimes 1\right) & =w_{A}\left(f_{A}\right) w_{B}^{\circ}(1)=w_{A}\left(f_{A}\right),
\end{aligned}
$$

entailing $\varphi_{n}\left(f_{A} \otimes 1\right)=w_{A}\left(f_{A}\right)$ for all $f_{A} \in C_{b}\left(X_{A}\right)$. Moreover, we have

$$
\varphi_{n+1}\left(1 \otimes f_{B}\right)=\tau\left(\varphi_{n}\right)\left(1 \otimes f_{B}\right) \quad\left(f_{B} \in C_{b}\left(X_{B}\right)\right)
$$

for all $n \in \mathbb{N}$.

Another sequence of states $w_{(N)}$ in $\mathscr{S}(N \in \mathbb{N})$ will then be defined from the $\varphi_{n}$ by an averaging procedure:

$$
w_{(N)}(f)=\frac{1}{N}\left(\sum_{n=1}^{N} \varphi_{n}(f)\right) \quad\left(f \in C_{b}(X)\right) .
$$

It then follows immediately from the properties of the $\varphi_{n}$ that

$$
w_{(N)}\left(f_{A} \otimes 1\right)=w_{A}\left(f_{A}\right) \quad\left(f_{A} \in C_{b}\left(X_{A}\right), N \in \mathbb{N}\right),
$$

and furthermore, using (3.3),

$$
\begin{aligned}
\left|\tau\left(w_{(N)}\right)\left(1 \otimes f_{B}\right)-w_{(N)}\left(1 \otimes f_{B}\right)\right| & =\left|\frac{1}{N}\left(\sum_{n=1}^{N} \tau\left(\varphi_{n}\right)\left(1 \otimes f_{B}\right)-\varphi_{n}\left(1 \otimes f_{B}\right)\right)\right| \\
& =\left|\frac{1}{N}\left(\varphi_{N+1}\left(1 \otimes f_{B}\right)-\varphi_{1}\left(1 \otimes f_{B}\right)\right)\right| \\
& \leq \frac{2}{N}|| f_{B} \|_{\infty} \quad\left(f_{B} \in C_{b}\left(X_{B}\right), \quad N \in \mathbb{N}\right)
\end{aligned}
$$

Owing to the Banach-Alaoglu theorem [28] that we have already mentioned in the previous section, there is a generalized sequence $\left\{N_{\kappa}\right\}_{\kappa \in \mathcal{K}}$, where $\mathcal{K}$ is some directed index set, such that $\lim _{\kappa} N_{\kappa}=\infty$ and such that

$$
\lim _{\kappa} w_{N_{\kappa}}(f)=w(f) \quad\left(f \in C_{b}(X)\right)
$$

for some state $w \in \mathscr{S}$. 
In view of (3.5) and (3.6), and observing the assumed continuity of $\tau$ which asserts that $\lim _{\kappa} \tau\left(w_{N_{\kappa}}\right)(f)=\tau\left(\lim _{\kappa} w_{N_{\kappa}}\right)(f)$ for all $f \in C_{b}(X)$, one now obtains that $w$ has the properties claimed in the statement above,

$$
\begin{aligned}
& w\left(f_{A} \otimes 1\right)=w_{A}\left(f_{A}\right) \quad\left(f_{A} \in C_{b}\left(X_{A}\right)\right) \quad \text { and } \\
& \tau(w)\left(1 \otimes f_{B}\right)=w\left(1 \otimes f_{B}\right) \quad\left(f_{B} \in C_{b}\left(X_{B}\right)\right) .
\end{aligned}
$$

This proves the Theorem.

For the remaining part of this section, we introduce some definitions, following [27].

Let $\mu$ be a probability measure on the Borel sets of a metric space $X$. The measure is called tight if for any given $\varepsilon>0$ there is a compact subset $K$ of $X$ such that $\mu(X \backslash K)<\varepsilon$.

Similarly, a sequence $\left\{\mu_{n}\right\}_{n \in \mathbb{N}}$ of probability measures defined on the Borel sets of a metric space $X$ is called tight if for any given $\varepsilon>0$ there is some compact subset $K$ of $X$ such that

$$
\sup _{n \in \mathbb{N}} \mu_{n}(X \backslash K)<\varepsilon .
$$

Let $X_{A}$ and $X_{B}$ are metric spaces, $X=X_{A} \times X_{B}$, and let $\mu$ be a probability measure on the Borel sets of $X$. Then one can define the marginals of $\mu$,

$$
\mu^{(A)}(O)=\mu\left(O \times X_{B}\right), \quad \mu^{(B)}(P)=\mu\left(X_{A} \times P\right)
$$

for Borel sets $O$ of $X_{A}$ and Borel sets $P$ of $X_{B}$. Both $\mu^{(A)}$ and $\mu^{(B)}$ are probability measures.

For later use, we put on record the following statement (see [27], Prob. 5.9).

Lemma 3.1 Let $X_{A}$ and $X_{B}$ be metric spaces and let $\left\{\mu_{n}\right\}_{n \in \mathbb{N}}$ be a sequence of probability measures on the Borel sets of $X=X_{A} \times X_{B}$.

Then $\left\{\mu_{n}\right\}_{n \in \mathbb{N}}$ is tight if and only if the sequences of marginals $\left\{\mu_{n}^{(A)}\right\}_{n \in \mathbb{N}}$ and $\left\{\mu_{n}^{(B)}\right\}_{n \in \mathbb{N}}$ are both tight.

We shall also make use of the following result.

Prohorov's Theorem [26, 27]

Suppose that $X$ is a locally compact metric space and that $\left\{\mu_{n}\right\}_{n \in \mathbb{N}}$ is a sequence of probability measures on the Borel sets of $X$.

If $\left\{\mu_{n}\right\}_{n \in \mathbb{N}}$ is tight, then it is weakly relatively compact: There are a probability measure $\mu$ on the Borel sets of $X$ and a subsequence $\left\{\mu_{n(k)}\right\}_{k \in \mathbb{N}}$ which converges weakly on $C_{b}(X)$ to $\mu$, i.e.

$$
\lim _{k \rightarrow \infty} \int_{X} f d \mu_{n(k)}=\int_{X} f d \mu \quad\left(f \in C_{b}(X)\right) .
$$


Theorem 3.2 Let $X=X_{A} \times X_{B}$ define a classical statistical bi-partite system where $X_{A}$ and $X_{B}$ are locally compact metric spaces.

Let $\tau: \mathscr{S}^{(P)} \rightarrow \mathscr{S}^{(P)}$ be an operation on the state space of $C_{b}(X)$ induced by probability measures on the Borel sets of $X$, and let $w_{A} \in \mathscr{S}_{A}^{(P)}$ be a state on $C_{b}\left(X_{A}\right)$ which is induced by a probability measure $\mu_{A}$ on the Borel sets of $X_{A}$, assumed to be tight.

Suppose also that there is a state $w_{B}^{\circ} \in \mathscr{S}_{B}^{(P)}$ on $C_{b}\left(X_{B}\right)$ which is induced by a probability measure $\mu_{B}^{\circ}$ on the Borel sets of $X_{B}$, with the property that the sequence of probability measures

$$
\mu_{B}^{(n)}(P)=\tau^{n}\left(\mu_{A} \times \mu_{B}^{\circ}\right)\left(X_{A} \times P\right) \quad(n \in \mathbb{N})
$$

on the Borel sets $P$ of $X_{B}$ is tight.

Then there is a state $w \in \mathscr{S}^{(P)}$ induced by a probability measure $\mu$ on the Borel sets of X such that

$$
\begin{aligned}
& w\left(f_{A} \otimes 1\right)=w_{A}\left(f_{A}\right) \quad\left(f_{A} \in C_{b}\left(X_{A}\right)\right) \quad \text { and } \\
& \tau(w)\left(1 \otimes f_{B}\right)=w\left(1 \otimes f_{B}\right) \quad\left(f_{B} \in C_{b}\left(X_{B}\right)\right) .
\end{aligned}
$$

Proof Using $w_{B}^{\circ}$, the sequence of states $\varphi_{n}$ and whence, the sequence of states $w_{(N)} \in \mathscr{S}^{(P)}(N \in \mathbb{N})$ is constructed as in the proof of Theorem 3.1. It follows easily from the assumptions that the states $w_{(N)}$ on $C_{b}(X)$ are indeed induced by probability measures, denoted $\mu_{(N)}$. We wish to show that the sequence $\left\{\mu_{(N)}\right\}_{N \in \mathbb{N}}$ is tight. According to Lemma 3.1, this follows once it is shown that the sequences of marginals $\left\{\mu_{(N)}^{(A)}\right\}_{N \in \mathbb{N}}$ and $\left\{\mu_{(N)}^{(B)}\right\}_{N \in \mathbb{N}}$ are both tight. Making use of (3.5), one can see that

$$
\mu_{(N)}^{(A)}=\mu_{A} \quad(N \in \mathbb{N})
$$

and as $\mu_{A}$ has been assumed to be tight, tightness follows for $\left\{\mu_{(N)}^{(A)}\right\}_{N \in \mathbb{N}}$. Similarly, (3.3) shows that

$$
\begin{gathered}
\mu_{(N)}^{(B)}(P)=\frac{1}{N} \sum_{n=1}^{N} \tau^{n}\left(\mu_{A} \times \mu_{B}^{\circ}\right)\left(X_{A} \times P\right) \\
=\frac{1}{N} \sum_{n=1}^{N} \mu_{B}^{(n)}(P)
\end{gathered}
$$

holds for all Borel sets $P$ of $X_{B}$. Since the sequence $\left\{\mu_{B}^{(n)}\right\}_{n \in \mathbb{N}}$ is by assumption tight, the same can easily be concluded for the averaged sequence $\left\{\mu_{(N)}^{(B)}\right\}_{N \in \mathbb{N}}$. Hence, the sequence of probability measures $\left\{\mu_{(N)}\right\}_{N \in \mathbb{N}}$ is tight. It can therefore be concluded from Prohorov's Theorem that there is a subsequence $\left\{\mu_{(N(k))}\right\}_{k \in \mathbb{N}}$ which converges weakly to a probability measure $\mu$ on the Borel sets of $X$. Then (3.12) follows from 
(3.14), and (3.13) is obtained using (3.6) in combination with the weak continuity of $\tau$ as in the final part of the proof of Theorem 3.1.

\section{A Simple Example-And Ergodicity}

The Cesáro-type limit which enters in the construction of the state $w$ fulfilling the D-CTC condition in Theorems 3.1 and 3.2 is very reminiscent of the discrete time-step evolution averaged limit which is a standard way of obtaining invariant states under a transformation. From this perspective, the construction of $w$ is related to Birkhoff's ergodic theorem [32, Theorem 5.1.1]. In this section, we elaborate a bit on this relation, considering a very simple example: the two-body problem with a spherically symmetric central potential coupling two point masses (particles) in Hamiltonian mechanics. Thus, we have $X_{A}=X_{B}=T^{*}\left(\mathbb{R}^{3}\right) \simeq \mathbb{R}^{3} \times \mathbb{R}^{3}$ as phase spaces for the particles labelled "A" and "B", with Hamiltonian function

$$
H_{\lambda}\left(q_{A}, p_{A} ; q_{B}, p_{B}\right)=\frac{1}{2 m_{A}}\left|p_{A}\right|^{2}+\frac{1}{2 m_{B}}\left|p_{B}\right|^{2}+V\left(\left|q_{A}-q_{B}\right|\right)+\lambda V_{\mathrm{ex}}\left(q_{A}, q_{B}\right)
$$

with particle masses $m_{A}$ and $m_{B}$, and $V: \mathbb{R}_{>0} \rightarrow \mathbb{R}$ a smooth function. $V_{\mathrm{ex}}\left(q_{A}, q_{B}\right)$ is an external potential and $\lambda \geq 0$ is a coupling constant. The coupling constant is introduced mainly to distinguish two cases: $\lambda=0$, i.e. the center-of-mass moves freely, and $\lambda=1$, where the center-of-mass moves under the influence of the external potential. We think of Newtonian-type potentials like $V(r)=-\alpha / r$ and $V_{\text {ex }}\left(q_{A}, q_{B}\right)=-\left(\beta_{A} /\left|q_{A}\right|+\beta_{B} /\left|q_{B}\right|\right)$ where $\alpha, \beta_{A}, \beta_{B}>0$; however more general (binding, central) potentials are also possible. For the Newtonian-like potentials, we would exclude configurations with $q_{A}=0, q_{B}=0$ and $q_{A}=-q_{B}$.

In the case of $\lambda=0$, the trajectories of bound states (excluding head-on collision) correspond to closed ellipses on which the particles travel in their configuration spaces around the center-of-mass as focal point. For more general binding potentials $V$, perihelion shifts may occur for bound states, so that the trajectories of the particles in their configuration spaces are rosettae revolving around the center-of-mass in a common orbital plane.

For $\lambda=1$, the trajectories of the bound states are approximately similar; however the center-of-mass trajectory is almost an ellipse with the center of the potential at $q_{A}=0$ and $q_{B}=0$ as focal point. This case corresponds to a planet (the "A"-system) with a moon or satellite (the "B" system) that are bound in the gravitational field of a very heavy central star which under the mutual gravitational interaction remains almost at rest and can therefore be effectively described as an external potential. (For this to be a good approximation, the stellar mass is to be very much larger than $m_{A}$ and $m_{B}$, and also $\left|q_{A}\right|$ and $\left|q_{B}\right|$ are to be very much larger than $\left|q_{A}-q_{B}\right|$.)

Then let $F_{t}: X_{A} \times X_{B} \rightarrow X_{A} \times X_{B}$ denote the phase flow map for the two-body system with the Hamiltonian function $H_{\lambda}$, taking phase space points from some "initial" time $t_{i}$ to some "final" time $t_{f}=t_{i}+t$. It induces the $C^{*}$-algebra isomorphism 


$$
A_{t} f=f \circ F_{t}^{-1} \quad\left(f \in C_{b}\left(X_{A} \times X_{B}\right)\right)
$$

on the phase space functions, and in turn it induces the operation

$$
\left(\tau_{t} w\right)(f)=w\left(A_{t} f\right)
$$

on the state space $\mathscr{S}$ of $C_{b}\left(X_{A} \times X_{B}\right)$. Note that one also has $\tau_{t}: \mathscr{S}^{(P)} \rightarrow \mathscr{S}^{(P)}$, i.e. it maps the set of probability measures on the Borel sets of $X=X_{A} \times X_{B}$ to itself.

We wish to illustrate the significance of the tightness assumption in Theorem 3.2. Let us look at phase space points $x_{A}=\left(q_{A, i}, p_{A, i}\right)$ and $x_{B}=\left(q_{B, i}, p_{B, i}\right)$ at "initial" time $t_{i}$. The points correspond to pure states on $C_{b}\left(X_{A}\right)$ and $C_{b}\left(X_{B}\right)$, induced by Diracmeasures $\delta_{x_{A}}$ and $\delta_{x_{B}}$ concentrated at $x_{A}$ and $x_{B}$, respectively. The configuration space points $q_{A}$ and $q_{B}$ correspond to the inertial coordinates of the particles.

Assume first that $\lambda=0$. Then, given any $x_{A}$, it is always possible to find an $x_{B}$ so that the resulting particle trajectories form a bound system, but in general, the center-of-mass will then move with a constant (non-zero) velocity. In this case one cannot expect that the sequence of measures ${ }^{5} \tau_{t}^{n}\left(\delta_{x_{A}} \times \delta_{x_{B}}\right)(n \in \mathbb{N})$ will be tight because (i) of the validity of Liouville's theorem and (ii) the support of these measures in the $q$-components remains within a ball of sufficiently large, fixed radius around the center of mass at time $n \cdot t$, so it moves to infinity as $n \rightarrow \infty$. (One could compensate that by re-defining $F_{t}$ so as to subtract the center-of-mass motion, but that re-definition depends on the choice of $x_{A}$ and $x_{B}$.)

Consider now the case $\lambda=1$. Then there are a non-empty open set $Y$ and a compact set $K$ in $X=X_{A} \times X_{B}$ so that $F_{t}^{n}(Y) \subset K$ for all $n \in \mathbb{N}$. Therefore, whenever one chooses a point $x_{A} \in X_{A}$ that is in the $A$-component of $Y$, there is some $x_{B}$ in $X_{B}$ with $\left(x_{A}, x_{B}\right) \in Y$. Consequently, one obtains that the sequence of marginal measures $P \mapsto \tau^{n}\left(\delta_{x_{A}} \times \delta_{x_{B}}\right)\left(X_{A} \times P\right)(n \in \mathbb{N})$ is tight because all of these measures have their support in the $B$-component projection of $K$, which is a compact subset of $X_{B}$. Hence, in this setting, we can apply Theorem 3.2. As already explained, $\tau$ is identified with $\tau_{t}$, and we may choose $w_{A}$ as being induced by a probability measure $\delta_{x_{A}}$ for a phase space point $x_{A}$ that is part of a bound state in $Y$ as just explained; there is actually a wide range of choice for such $x_{A}$. Then we may choose $w_{B}^{\circ}$ as any $\delta_{x_{B}}$ so that $\left(x_{A}, x_{B}\right) \in Y$. As discussed, the assumptions of Theorem 3.2 are fulfilled, and there is a state $w$ given by a probability measure on the Borel sets of $X$ so that the properties (3.12) and (3.13) are fulfilled. On the other hand, if one chooses $\delta_{x_{A}}$ for $w_{A}$ as before, but selects as $w_{B}^{\circ}$ a $\delta_{x_{B}}$ so that $\left(x_{A}, x_{B}\right)$ does not correspond to a bound state, one cannot expect that the required tightness assumption is fulfilled, by a reasoning similar to the $\lambda=0$ case before.

In the case $\lambda=1$ and $\left(x_{A}, x_{B}\right) \in Y$, let us try to understand the properties of the state $w$ constructed in Theorem 3.2 with the properties (3.12) and (3.13). To this end, to ease the illustration, we consider a very much simplified situation. We assume that $m_{A}$ is very much larger than $m_{B}$ so that the motion of the "A" particle

\footnotetext{
${ }^{5}$ Here we slightly abuse notation and identify states with the measures by which they are induced; instead of $\tau_{t}^{n}\left(\delta_{x_{A}} \times \delta_{x_{B}}\right)$ we should write, more correctly, the transformed measures $\left(\delta_{x_{A}} \times \delta_{x_{B}}\right) \circ F_{t}^{n}$.
} 
coincides to very good approximation with the center-of-mass motion. Furthermore, we assume that $\left|q_{A}\right|$ is extremely large so that, even for a very high number of orbits of the " $\mathrm{B}$ " particle around the " $\mathrm{A}$ " particle, the center-of-mass motion is approximately a free motion. This corresponds to a satellite, or "piece of rock" ("B" particle) orbiting a planet ("A" particle) which is on a very remote orbit around a star. We assume that the orbital planes are coincident, and that the orbit of the satellite around the planet is, to good approximation, circular. Then we consider the measures (3.15) constructed in Theorem 3.2 for the present situation,

$$
\mu_{(N)}^{(B)}(P)=\frac{1}{N} \sum_{n=1}^{N} \tau_{t}^{n}\left(\delta_{x_{A}} \times \delta_{x_{B}}\right)\left(X_{A} \times P\right) .
$$

and change to inertial coordinates $q_{A}, q_{B}$ in which (according to our simplifying assumptions) the planet is approximately at rest. The measures $\mu_{(N)}^{(B)}$ depend, of course, on how $t$ is chosen. For the assumed (approximately) circular orbit, let $T$ denote the orbital period. There are several cases that one can consider:

(i) $t=k T$ for some $k \in \mathbb{N}$. Then $\tau_{t}^{n}\left(\delta_{x_{A}} \times \delta_{x_{B}}\right)\left(X_{A} \times P\right)$ is independent of $n$ for all $n \in \mathbb{N}$ since we have (in our approximation) $F_{t}\left(x_{A}, x_{B}\right)=\left(x_{A}, x_{B}\right)$ in this case. Therefore, $\mu_{(N)}^{(B)}=\left(\delta_{x_{A}} \times \delta_{x_{B}}\right)\left(X_{A} \times P\right)$ is also independent of $N$ : Applying $\tau_{t}$ just reproduces the initial phase space points.

(ii) $t=k T / \ell$ for some $k, \ell \in \mathbb{N}$. Then $F_{t}^{\ell}\left(x_{A}, x_{B}\right)=\left(x_{A}, x_{B}\right)$ and hence $\tau_{t}\left(\sum_{j=1}^{\ell}\left(\delta_{x_{A}} \times \delta_{x_{B}}\right)\left(X_{A} \times P\right)=\sum_{j=1}^{\ell} \tau_{t}^{j}\left(\delta_{x_{A}} \times \delta_{x_{B}}^{t}\right)\left(X_{A} \times P\right)\right.$. Therefore, $\mu_{(m \cdot \ell)}^{(B)}(P) \stackrel{x_{A}}{=} \mu_{(\ell)}^{(B)}(P)$ for all $m \in \mathbb{N}$.

Thus, in case (i), there is a state $w$ fulfilling (3.12) and (3.13) whose partial state on the "B"-part of the system, at initial time $t_{i}$, is given just by $\delta_{x_{B}}$. In case (ii), there is a state $w$ fulfilling (3.12) and (3.13) with partial state on the "B"-part given by

$$
\frac{1}{\ell} \sum_{j=1}^{\ell} \delta_{x_{B}(j k T / \ell)}
$$

where $\left(x_{A}, x_{B}\left(t^{\prime}\right)\right)=F_{t^{\prime}}\left(x_{A}, x_{B}\right)$. This "statistical mixture of phase space points" can be represented as $\ell$ copies of the $B$-particle, i.e. $\ell$ identical satellites, each on the same circular orbit, separated in position and momenta by a $1 / \ell$ fraction of the orbit, so that this phase space distribution gets mapped to itself under the phase space map $F_{k T / \ell}^{\ell}$. Quite clearly, the cases (i) and (ii) correspond to periodic orbits of the (effective) motion of the satellite around the planet.

(iii) $t=r T$ with $r$ irrational. In this case, there are no "periods" in the sequence $\mu_{(N)}^{(B)}(N \in \mathbb{N})$ and thus any state $w$ constructed in the proof of Theorem 3.2 with the properties (3.12) and (3.13) can be deduced to be induced, on the "B"-part of the system, by a measure $P \mapsto \mu\left(X_{A} \times P\right)$ which is supported on a dense set on the circular orbit of the satellite around the planet. This follows since on the circle, the irrational rotations (1) are known to produce dense orbits under 

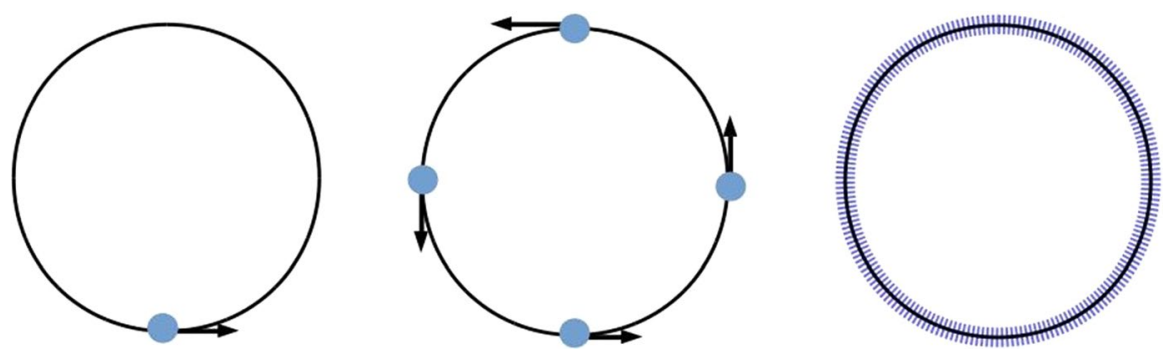

Fig. 2 Illustration of the three cases (i), (ii) and (iii) for the distribution of the state $w$ on the "B"-part of the system at initial time $t_{i}$ mentioned in the text, from left to right. For case (i), the initial position $q_{B, i}$ of the "satellite" on the circular orbit is depicted by the blue dot and its initial momentum $p_{B, i}$ is represented by the arrow. Case (ii) shows a distribution for $k=4$, with four copies of the "satellite" separated by moving the phase space points by $T / 4$ on the orbit. In case (iii), one obtains a dense distribution over the orbit; the momenta are not indicated (Color figure online)

successive applications by a classic result of Kronecker [32, Theorem 3.2.3] and they (2) are ergodic with respect to the Lebesgue-measure on the the circle [32, Theorem 3.5.7].

The three cases are illustrated in Fig. 2. For an interactive illustration, see [33]

Thus, under the very idealized assumptions made for the simplified situation as described, there is actually a state $w$ fulfilling the D-CTC condition for all $F_{t}$ $(t \in \mathbb{R})$ : The ergodic state, obtained by the construction of $w$ in Theorem 3.1 for the case that $\tau=\tau_{t^{\prime}}$ induced by $F_{t^{\prime}}$ for any $t^{\prime}=r T$ with $r$ irrational. This amounts to taking the Lebesgue measure on the circular trajectory of the satellite. It is worth pointing out that in [3] (Appendix B), solutions to the D-CTC problem in a more general formal framework are also obtained by means of an ergodic averaging.

Going back to the days of the very inception of ideas on ergodicity, when thinking of a satellite orbiting a planet, the rings of the planet Saturn are an example that one might envisage as an approximate realization of the ergodic state. (This "example" appears in publications of Boltzmann, see [34] for references and discussion.) Indeed, if one evolves the ring system by an arbitrary time-step, it appears unchanged, at least at scales larger than about $10 \mathrm{~km}$ which is tiny compared to the dimensions of the ring orbits - at scales larger than around $10 \mathrm{~km}$, the rings, which are mostly formed by rocks and pieces of ice of various sizes between the millimeter and kilometer scale, appear almost homogeneous in the angular direction (while there are significant density variations in the radial direction) [35]. (It should be noted that the dynamics of the rings of Saturn is only approximately ergodic, see e.g. $[35,36]$ and literature cited there for investigations on this problem.) However, our discussion in this section should serve to illustrate that the D-CTC condition is nothing extraordinary in classical statistical mechanics, that it relates to ergodicity and can be viewed as approximately realized in macroscopic physical systems at appropriate scales. 


\section{Discussion and Conclusion}

We have shown that the D-CTC condition is generically fulfilled for classical statistical bi-partite systems, under very general (yet mathematically precise) conditions. The D-CTC condition originated from Deutsch's proposal for giving a description of what it means that parts of quantum systems undergo processes that might be viewed as analogues to "going backwards in time". However, whether or not that condition can be fulfilled rests mainly on convexity and completeness of the space of states of a system, irrespective of its quantum or classical nature. Moreover, in the light of the results of [16], as we have indicated in Sect. 1, one might also doubt if the D-CTC condition actually says very much about closed timelike curves as they are understood in general relativity. This may call into question if the D-CTC condition really is a means of "treating time travel quantum mechanically" (title of the article [11]) or if statements like "quantum mechanics therefore allows for causality violation without paradoxes whilst remaining consistent with relativity" [37] are well-founded.

The starting point of Deutsch's discussion was a classical system where the states consist of finite sequences of "bits", i.e. the state space is a discrete, finite set, not admitting convex combinations. That assumption restricts the choice of states fulfilling the D-CTC considerably, as is shown in an example in [1]. In contrast, taking "q-bits" as the "quantized" version of a classical "bit" system naturally renders a convex and complete state space so that the D-CTC problem generically has many solutions. Nevertheless, it is rather the possibility to take classical statistical mixtures of states than anything specifically quantum mechanical that warrants solutions to the D-CTC condition in a q-bit system. Allowing classical statistical mixtures of "bit" states would have the same effect to this end. (However, in applications, "bit" states are introduced exactly for the purpose of avoiding uncertainties in state discrimination that may occur e.g. in the form of classical mixtures of states, so that from that perspective, forming statistical mixtures doesn't appear natural for "bit" state systems. Yet it is a viable theoretical possibility.)

Therefore, one should be careful not to jump to explanations as to why the D-CTC condition is fulfilled in quantum systems which rely on typical features of quantum systems or their behaviour in spacetime (e.g. interference or uncertainty relations) as this does not relate to what the D-CTC condition-or the fact that it can be generically fulfilled-is based on; such explanations may result in inadequate interpretations and are therefore misleading. We would regard the attempt in [1] to give an explanation for the solvability of the D-CTC problem in quantum mechanics based on a many-worlds interpretation, in this sense, as unconvincing (it has elsewhere been criticized on other grounds [38]).

Yet, the fact that the D-CTC condition can generically be solved in q-bit systems can open interesting aspects for quantum computing and quantum communication [1, $2,10-15]$. In this context, the central point of investigation is-using the notation of the beginning part of Sect. 1-the question what "output" states on the "A" part of the bi-partite system,

$$
\langle\mathbf{a}\rangle_{\tilde{A}}=\operatorname{Tr}_{A}\left(\tilde{\varrho}_{A} \mathbf{a}\right)=\operatorname{Tr}\left(\rho U^{*}(\mathbf{a} \otimes \mathbf{1}) U\right),
$$


can be derived from the density matrix $\varrho$ fulfilling the D-CTC condition for a given unitary $U$ on the full system, and an "input" state $\langle\mathbf{a}\rangle_{A}=\operatorname{Tr}_{A}\left(\varrho_{A} \mathbf{a}\right)$ on the "A" part of the system. In other words, the investigation is on the map $\phi: \varrho_{A} \mapsto \tilde{\varrho}_{A}$ for given $U$. There are some difficulties here. First, since $\rho$ is not uniquely determined by $U$ and $\varrho_{A}, \phi$ is not naturally defined as a map on the state space of the "A" part of the bi-partite quantum mechanical system. Secondly, given that a map $\phi$ can be determined by imposing additional selection criteria, if $\varrho$ is constructed as in [1], then $\phi$ fails to be convex, i.e. it isn't an operation. This is also to be expected in the classical (measure-theoretic) framework which we have considered in the present article in the sense that in general, the dependence of the state $w$ in the proof of Theorems 3.1 and 3.2 and of the partial state $\tilde{w}_{A}\left(f_{A}\right)=\tau(w)\left(f_{A} \otimes 1\right)$ is not convex in the given state $w_{A}$ on the "A"-part of the system.

That failure of $\phi$ to be convex in $\varrho_{A}$ is in the literature usually referred to by saying that (solutions to the) D-CTC condition are "non-linear" in the input state $\varrho_{A}$, and it has been discussed that this may impede the utility of the solvability of the D-CTC problem for the purposes of quantum computing. For considerable further investigation on this issue, see again the articles just cited, and also references given there. A contention expressed in [2] is that due to the failure of $\phi$ to be convex, the D-CTC condition is incomplete. Basically, that is also our conclusion, however potentially at a more fundamental level, in the sense that the D-CTC condition doesn't depend on typically quantum mechanical features of a bi-partite system. When claiming that quantum mechanics is an important ingredient in avoiding the notorious paradoxes of time travel, but then implementing that formally at the level of the D-CTC condition which is not sensitive to whether a bi-partite system is of quantum mechanical nature or not, and instead just depends on its basic statistical properties, something seems to be missing.

Concerning the possibility that the D-CTC condition isn't sufficiently complete or specific to really allow statements connecting quantum processes and closed timelike curves, we have commented in [16] that a possible approach could be to include spacetime localization into the description, in the spirit of the algebraic framework of quantum field theory as sketched in Sect. 1. Still, one would have to connect locality properties of the unitary operator $U$, or of the operation $\tau$ with the locality properties of the observables. In the present paper, we have not considered locality properties of the observables and that is, in a certain sense, an omission. Therefore, it would be interesting to see if, and how, our results might extend to the analysis of billiard ball collisions in the presence of wormhole-type time machines [5-7].

In [17] we have pointed out that the results in [16] (as well as in [17]) on whether the D-CTC condition can be fulfilled or not depend very much on the assumptions made, and on whether one insists that the D-CTC condition is fulfilled exactly, or just approximately (to arbitrary precision). In fact the question of the adequate mathematical idealization is a common problem when trying to explore unchartered territory in physics by theoretical methods. In the context of the question if one may ascribe physical reality to anything which one might bear the properties of a "time machine", i.e. processes which can be interpreted as brought about by the presence of closed timelike curves, the problem of what constitutes an adequate mathematical idealization acquires considerable importance, and we think that one of the inspiring 
aspects about the investigation of the D-CTC condition is to highlight that issue, and potentially gain some insight.

Acknowledgements The authors would like to thank Christopher J. Fewster for comments on the manuscript.

Funding Open Access funding enabled and organized by Projekt DEAL.

Open Access This article is licensed under a Creative Commons Attribution 4.0 International License, which permits use, sharing, adaptation, distribution and reproduction in any medium or format, as long as you give appropriate credit to the original author(s) and the source, provide a link to the Creative Commons licence, and indicate if changes were made. The images or other third party material in this article are included in the article's Creative Commons licence, unless indicated otherwise in a credit line to the material. If material is not included in the article's Creative Commons licence and your intended use is not permitted by statutory regulation or exceeds the permitted use, you will need to obtain permission directly from the copyright holder. To view a copy of this licence, visit http://creativecommons.org/licen ses/by/4.0/.

\section{References}

1. Deutsch, D.: Quantum mechanics near closed timelike lines. Phys. Rev. D 44, 3197 (1991)

2. Brun, T.A., Wilde, M.M., Winter, A.: Quantum state cloning using Deutschian closed timelike curves. Phys. Rev. Lett. 111, 190401 (2013)

3. Pinzani, N., Gogioso, S., Coecke, B.: Categorical semantics for time travel. In: 34th Annual ACM/ IEEE Symposium on Logic in Computer Science, LICS 2019, Vancouver, BC, Canada, 24-27 June 2019, pp. 1-20 (2019). https://doi.org/10.1109/LICS.2019.8785664

4. Arntzenius, F., Maudlin, T.: Time travel and modern physics. Royal Inst. Philos. Suppl. 50, 169-200 (2002); revised online version (dated 2009) at Stanford Encyclopedia of Philosophy. https://plato. stanford.edu/entries/time-travel-phys/

5. Frolov, V.P., Novikov, I.D.: Physical effects in wormhole and time machine. Phys. Rev. D 42, 10571065 (1990)

6. Echeverria, F., Klinkhammer, G., Thorne, K.S.: Billiard balls in wormhole spacetimes with closed timelike curves: classical theory. Phys. Rev. D 44, 1077-1099 (1991)

7. Novikov, I.D.: Time machine and selfconsistent evolution in problems with selfinteraction. Phys. Rev. D 45, 1989-1994 (1992)

8. Deutsch, D., Lockwood, M.: The quantum physics of time travel. Sci. Am. 270(3), 68-74 (1994)

9. Avengers: Endgame, Cinema movie (the citation refers to a scene between 33:00 to 35:00 minutes into the running time). Marvel Studios (2019) (see https://en.wikipedia.org/wiki/Avengers:_Endga me)

10. Ahn, D., Myers, C.R., Ralph, T.C., Mann, R.B.: Quantum state cloning in the presence of a closed timelike curve. Phys. Rev. A 88, 022332 (2013)

11. Allen, J.M.A.: Treating time travel quantum mechanically. Phys. Rev. A 90, 042107 (2014)

12. Brun, T.A., Wilde, M.M.: Simulations of closed timelike curves. Found. Phys. 47, 375-391 (2017)

13. Ralph, T.C., Myers, C.R.: Information flow of quantum states interacting with closed timelike curves. Phys. Rev. A 82, 062330 (2010)

14. Bub, J., Stairs, A.: Quantum interactions with closed timelike curves and superluminal signalling. Phys. Rev. A 89, 022311 (2014)

15. Bennett, C., Leung, D., Smith, G., Smolin, J.: Can closed timelike curves or non-linear quantum mechanics improve quantum state discrimination or help solve hard problems? Phys. Rev. Lett. 103, 170502 (2009)

16. Tolksdorf, J., Verch, R.: Quantum physics, fields and closed timelike curves: the D-CTC condition in quantum field theory. Commun. Math. Phys. 357, 319-351 (2018)

17. Verch, R.: The D-CTC condition in quantum field theory. In: Finster, F., Giulini, D., Kleiner, J., Tolksdorf, J. (eds.) Progress and Visions in Quantum Theory in View of Gravity, pp. 221-232. Birkhäuser, Cham (2020) 
18. Haag, R.: Local Quantum Physics, 2nd edn. Springer, Berlin (1996)

19. Summers, S.J.: On the independence of local algebras in quantum field theory. Rev. Math. Phys. 2 , 201-247 (1990)

20. Fewster, C.J.: The split property for locally covariant quantum field theories in curved spacetime. Lett. Math. Phys. 105(12), 1633-1661 (2015)

21. Verch, R., Werner, R.F.: Distillability and positivity of partial transposes in general quantum field systems. Rev. Math. Phys. 17, 545-576 (2005)

22. Landsman, N.P.: Foundations of Quantum Theory. FTPH, vol. 188, Springer, Berlin (2017). https:// doi.org/10.1007/978-3-319-51777-3

23. Figueroa, H., Gracia-Bondía, J.M., Várilly, J.: Elements of Noncommutative Geometry. Birkhäuser Advanced Texts. Springer, New York (2001)

24. Pedersen, G.K.: $C^{*}$-Algebras and Their Automorphism Groups. Academic Press, New York (1979)

25. Hewitt, E., Stromberg, K.: Real and Abstract Analysis. Springer, Berlin (1975)

26. Prohorov, Y.V.: Convergence of random processes and limit theorems in probability theory. Theory Probab. Appl. 1, 157-214 (1956)

27. Billingsley, P.: Convergence of Probability Measures, 2nd edn. Wiley, New York (1999)

28. Reed, M., Simon. B.: Methods of Modern Mathematical Physics, vol. 1. Academic Press, New York (1975)

29. Gelfand, I., Naimark, M.: On the imbedding of normed rings into the ring of operators on a Hilbert space. Rec. Math. [Mat. Sbornik] N.S. 12, 197-217 (1943)

30. Mackey, G.W.: The Mathematical Foundations of Quantum Mechanics. W.A. Benjamin, New York (1963)

31. Abraham, R., Marsden, J.E.: Foundations of Mechanics, 2nd edn. Addison-Wesley, Boston (1978)

32. Silva, C.E.: Invitation to Ergodic Theory. Student Mathematical Library, vol. 42. American Mathematical Society, Providence (2007)

33. Kuehn, C.: An introduction to rotation theory. Online tutorial. http://tutorials.siam.org/dsweb/circl emaps/circle.pdf

34. Gallavotti, G.: Ergodicity: a historical perspective. Equilibrium and nonequilibrium. Eur. Phys. J. H41, 181-259 (2016)

35. Esposito, L.W.: Planetary Rings-A Post-Equinox View, 2nd edn. Cambridge University Press, Cambridge (2014)

36. Bodrova, A., Chechkin, A.V., Cherstvy, A., Metzler, R.: Quantifying non-ergodic dynamics of force-free granular gases. Phys. Chem. Chem. Phys. 17, 21791 (2015)

37. Ringbauer, M., Broome, M.A., Myers, C.R., White, A.G., Ralph, T.C.: Experimental simulation of closed timelike curves. Nat. Commun. 5, 4145 (2014)

38. Dunlap, L.: The metaphysics of D-CTCs: on the underlying assumptions of Deutsch's quantum solution to the paradoxes of time travel. Stud. Hist. Philos. Mod. Phys. 56, 39-47 (2016)

Publisher's Note Springer Nature remains neutral with regard to jurisdictional claims in published maps and institutional affiliations. 